\title{
Spontaneous Large Serous Retinal Pigment Epithelial Tear
}

\author{
Voraporn Chaikitmongkol Direk Patikulsila \\ Janejit Choovuthayakorn Nimitr Ittipunkul \\ Paradee Kunavisarut
}

Department of Ophthalmology, Faculty of Medicine, Chiang Mai University, Chiang Mai, Thailand

\section{Key Words}

Retinal pigment epithelial tear · Pigment epithelial detachment $\cdot$ Retinal pigment epithelial rip

\begin{abstract}
Purpose: To report cases of spontaneous retinal pigment epithelial (RPE) tear complicating serous pigment epithelial detachment (PED).

Methods: The records of 3 Asian patients with spontaneous giant RPE tear were reviewed retrospectively by including clinical presentation, angiography, optical coherence tomography, fundus autofluorescence imaging, and visual outcome.
\end{abstract}

Results: Three patients (4 eyes) were included in this study, with a mean age of 48.3 (42-56) years, and a mean follow-up period of 7.75 (4-18) months. Fundus examination in all patients showed giant RPE tear associated with bullous PED. Two cases had a history of prior corticosteroid use, and 1 had no history of medication use. All 3 patients developed spontaneous resolution of subretinal fluid with no treatment. However, in patients who used corticosteroids, initial progression of the tear and subretinal fluid were observed despite ceasing medication. On subsequent follow-up, an incomplete RPE regeneration was demonstrated by fundus autofluorescence imaging, and choroidal neovascularization developed in 1 patient.

Conclusion: Large PED with RPE tear is a rare manifestation. When the fovea is spared, visual prognosis is favorable. No specific treatment is required, but careful choroidal neovascularization monitoring should be performed.

\section{Introduction}

Spontaneous large retinal pigment epithelial (RPE) tear is an unusual manifestation most commonly described in association with choroidal neovascularization (CNV) from 
age-related macular degeneration [1]. However, other causes have been reported, including central serous chorioretinopathy and post trauma [2-5]. Up until now, the natural course, predictive factors, and visual outcome of such cases have not been well characterized.

By using fundus imaging, optical coherence tomography (OCT), and fundus autofluorescence (FAF) imaging, this study reports 3 patients who developed serous pigment epithelial detachment (PED), which was complicated by giant RPE tear. Of the 3 patients, 2 (3 eyes) had a history of systemic corticosteroid use.

\section{Case Reports}

\section{Case 1}

A 42-year-old man presented a left eye with decreased vision, which he had had for 2 months. He had been treated for Bell's palsy with oral corticosteroid for 3 months prior to presenting his visual symptom. His best-corrected visual acuity (BCVA) was $6 / 6$ and 6/60 in his right and left eye, respectively. Fundus biomicroscopy of the left eye revealed bullous RPE detachment accompanied by a large RPE tear, which had a massive subretinal fibrin along the temporal margin in the posterior pole (fig. 1a) and exudative retinal detachment in the inferior peripheral fundus. The right eye had a small RPE detachment in the superotemporal parafoveal area. Fluorescein angiography (FA) of the left eye showed early hyperfluorescence of the bared RPE area, multiple hyperfluorescent leakage dots at the margin of the RPE tear, dye pooling into the RPE detachment and inferior serous retinal detachment in the late phase (fig. 1b). OCT of the left eye clearly demonstrated the presence of an RPE tear (fig. 1c). Despite ceasing corticosteroid use, the serous detachment continued to progress during the first 3 weeks. One month later, subretinal fluid gradually decreased and completely resolved 3 months after the first visit (fig. 1d). Visual acuity improved to 6/9 in the left eye and remained stable over the 6-month follow-up period, with no recurrence of PED.

Case 2

A 47-year-old woman presented with a 2-week history of visual disturbances in her left eye. She had been treated for lupus nephritis with oral prednisolone for 2 months prior to presentation. Her BCVA was 6/12 and 6/60 in her right and left eye, respectively. Biomicroscopic examination in the right and left eye revealed a large serous RPE detachment with a thin crescent-shaped RPE tear along the inferior border over the posterior pole ( $\underline{\text { fig. } 2 \mathrm{a}}$ ) and large RPE tear with overlying subretinal fluid (fig. 2b), respectively. FA revealed that both eyes had early demarcated hyperfluorescence corresponding to the RPE tear, hypofluorescence that corresponded to the retracted RPE edge, and gradual pooling into the PED area and subretinal space, with no other leakages observed (fig. 2c, d). Focal choroidal hyperpermeability was evidenced by indocyanine green angiography, superotemporal to the maculae; and presence of serous RPE detachment and tear was confirmed by OCT (fig. 2e, f) in both eyes. After examination, corticosteroid use was discontinued and the patient switched to other immunosuppressive drugs. During the first 2 weeks, subretinal fluid and RPE tear increased slightly, but gradually resolved and completely resorbed after 2 months (fig. $2 \mathrm{~g}$ ). At that time, vision improved to 6/9 in the right eye with foveal sparing, but decreased to counting finger in the left eye with foveal involvement. At 1-year follow-up, OCT showed incomplete repopulation of the RPE layer, and FAF imaging showed variation in the degree of fluorescence at the margin of the tear, and there was no recurrence of RPE detachment in either eye (fig. $2 \mathrm{~h}$, i).

Case 3

A 56-year-old man with an unremarkable medical history presented with 10 days of metamorphopsia and decreased central vision in his right eye. His BCVA was 6/24 and 6/6 in his right and left eye, respectively. Right fundus examination revealed a $360^{\circ} \mathrm{RPE}$ tear, with retracted RPE tissue located in the center of the serous detachment (fig. 3a), over the posterior pole. The left eye showed a small RPE detachment superonasal to the fovea. FA demonstrated hyperfluorescent characteristics of an RPE tear and pooling of subretinal fluid, and the OCT disclosed torn retinal pigment epithelium attached to the outer surface of the neurosensory retina (fig. $3 \mathrm{~b}$ ). Two weeks later, the patient's BCVA decreased to $6 / 60$ in the right eye, with persistent shallow subretinal fluid 
and further contraction of RPE tissue (fig. 2c). The fluid resolved slowly but clearly over the next 7 months. At 11-month follow-up, intraretinal exudates and hemorrhage developed (fig. 3d) at the centrally retracted RPE area, where FA showed a leakage of dye and OCT confirmed the presence of intraretinal fluid (fig. 3e). FAF imaging showed granular hyperfluorescence at the margin of the bare retinal pigment epithelium (fig. 3f).

\section{Discussion}

The exact pathophysiologic factors predisposing the patient to RPE tear remain uncertain. Nevertheless, tractional and shearing forces have been proposed as the underlying pathogenesis in some cases of $\mathrm{CNV}$ and trauma, while alteration in the function of the choriocapillaris-Bruch's membrane-RPE complex resulting in accumulation of the sub-RPE fluid had been assumed in the rest [6].

This report described 3 patients with spontaneous giant RPE tears complicating serous PED. Two of the patients had bilateral presentation and were associated with corticosteroid use. The other one (case 3) had unilateral presentation with none of the risk factors. This implies that corticosteroids may be an accelerating factor rather than a direct cause of RPE tear. After discontinuation of corticosteroid use in case 1 and 2, serous detachment increased initially within the first 2 weeks, but resolved rapidly in 2 months. On the other hand, resolution of the detachment was observed from the start and eventually resolved after 7 months of follow-up in the patient who did not use corticosteroids. From OCT images, no tractional signs of the vitreoretinal interface or any of the intraretinal structures were observed. Then, massive hydrostatic pressure beneath the retinal pigment epithelium could lead to large RPE tears in these cases.

Corticosteroids, either by exogenous administration or endogenous secretion, also have been implicated as a potential risk factor for developing or exacerbating acute rapid exudative macular and chronic bullous exudative retinal detachment $[7,8]$. Jampol et al. [9] postulated that different effects of posttranslational corticosteroids in each patient could possibly lead to a varied response of choroidal vascular and RPE modulation, with or without a history of receiving corticosteroids. In the event of RPE tears with foveal sparing, resolution of serous detachment has been reported with favorable visual outcomes $[4,7,10]$. Our patients with extrafoveal tears also showed good visual recovery, which is consistent with previous reports.

RPE healing is hypothesized by several mechanisms. Peiretti et al. [11] demonstrated the repopulation of torn retinal pigment epithelium, which was documented with OCT and FAF imaging. In 1 of the cases described here, RPE regeneration was also detected over the tear by color fundus photography, OCT, and FAF even though the regeneration was not complete at the end of follow-up. FAF of this patient revealed irregularity of hyperfluorescence at the margin of tears, which was postulated as decreased RPE cells containing lipofuscin, as well as central hypofluorescence corresponding to the area of absence in RPE cells.

CNV complicating RPE tear is a rare condition. CNV was detected 11 months following RPE tear in 1 of our reported cases with no history of corticosteroid use, making it plausible that $\mathrm{CNV}$ occurred secondarily and did not contribute to the initial tear. 
In summary, this report presented unusual cases of spontaneous tear of large serous PED with spontaneous resolution and no evidence of recurrence. One eye showed development of CNV during follow-up. Advanced technology, including angiography, OCT, and FAF, is useful in differentiating between diagnoses or confirming the correct diagnosis and following the natural course of diseases. Although there is no specific treatment for RPE tear, eyes with this condition should be followed closely, so that proper management can be granted in the event of complications such as CNV.

\section{Conclusion}

RPE tear complicating serous PED is an unusual manifestation. Good visual recovery is observed in cases of foveal sparing with spontaneous resolution. CNV is a rare complication that may develop later.

\section{Disclosure Statement}

The authors declare that they have no conflicts of interest. 


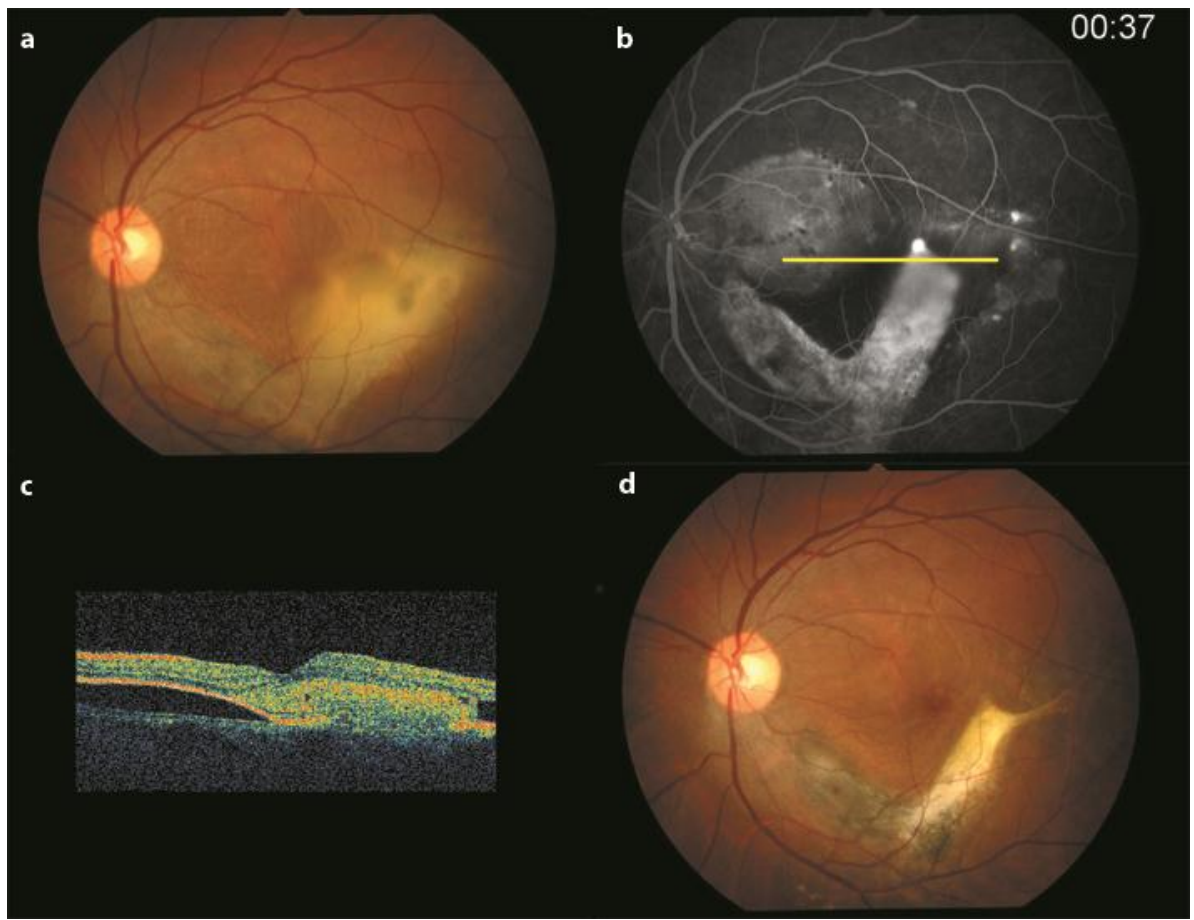

Fig. 1. Case 1. a Fundus photograph shows a V-shaped RPE tear and adjacent yellowish subretinal fibrinous exudates. b FA shows a window defect hyperfluorescence over the bared RPE area, with multiple leakage points along the temporal side of the RPE tear and dye pooling into the PED. The yellow line indicates the area scanned by OCT. c OCT demonstrates subretinal fibrin over the RPE disruption within an RPE detachment. $\mathbf{d}$ Fundus photography shows resolution of subretinal fluid and development of subretinal fibrosis in the previous fibrin deposition area after 6-month followup. 


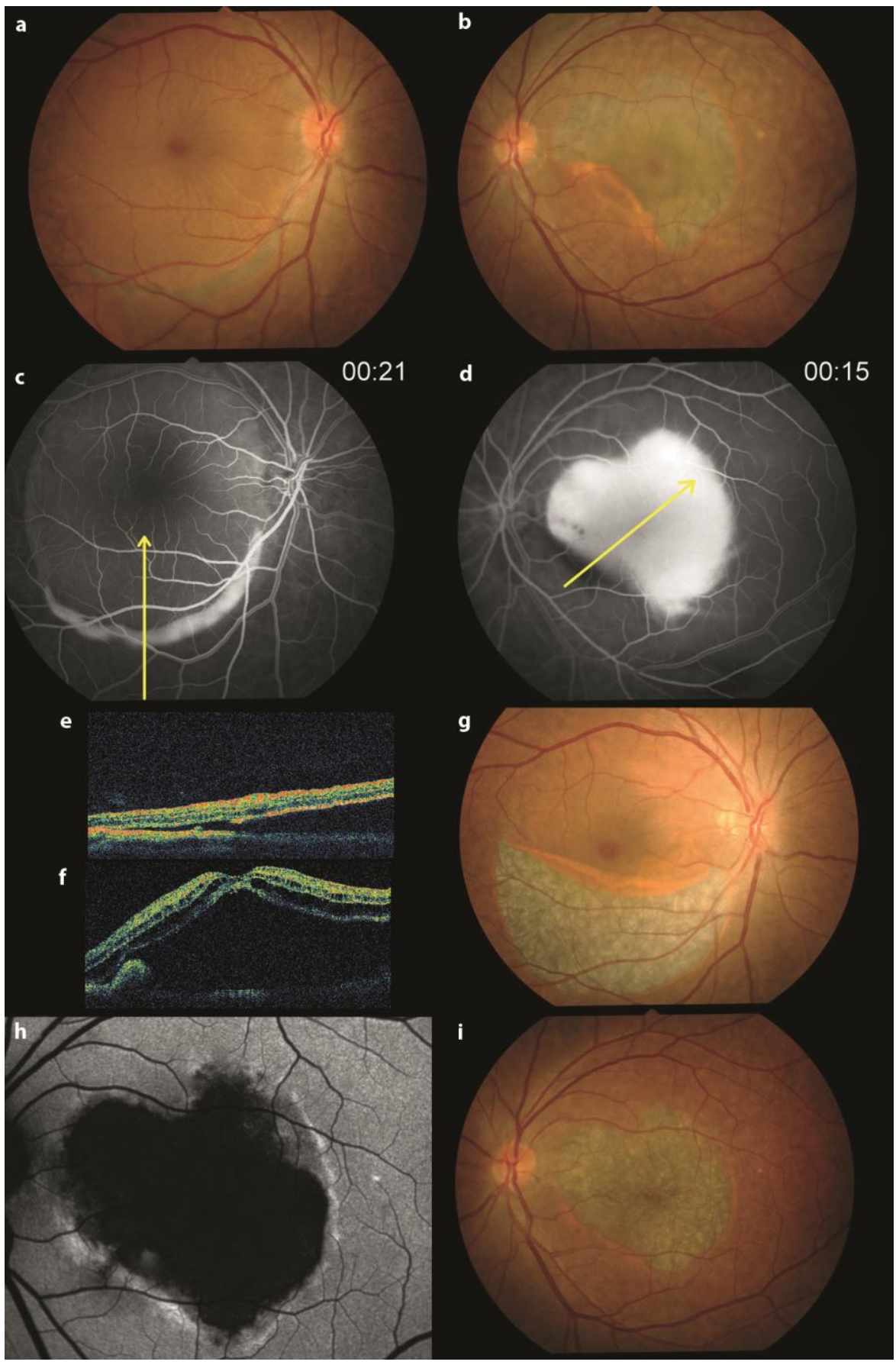

Fig. 2. Case 2. a, b Fundus photography illustrates serous RPE detachment and RPE tear in both eyes. c, $\mathbf{d}$ FA shows transmission hyperfluorescence corresponding to the area of absent retinal pigment epithelium, which is adjacent to an area of hypofluorescence that corresponds to the area of retracted retinal pigment epithelium. The yellow line indicates the area scanned by OCT. e, f OCT demonstrates disruption of the RPE layer corresponding to the tear. $\mathbf{g}$ Fundus photography shows complete resolution of subretinal fluid in the right eye at 2 -month follow-up. $\mathbf{h}$, i Color fundus photography demonstrates irregularity of the torn RPE edge corresponding to the stippled hyperfluorescent area of FAF imaging at 1-year follow-up. 


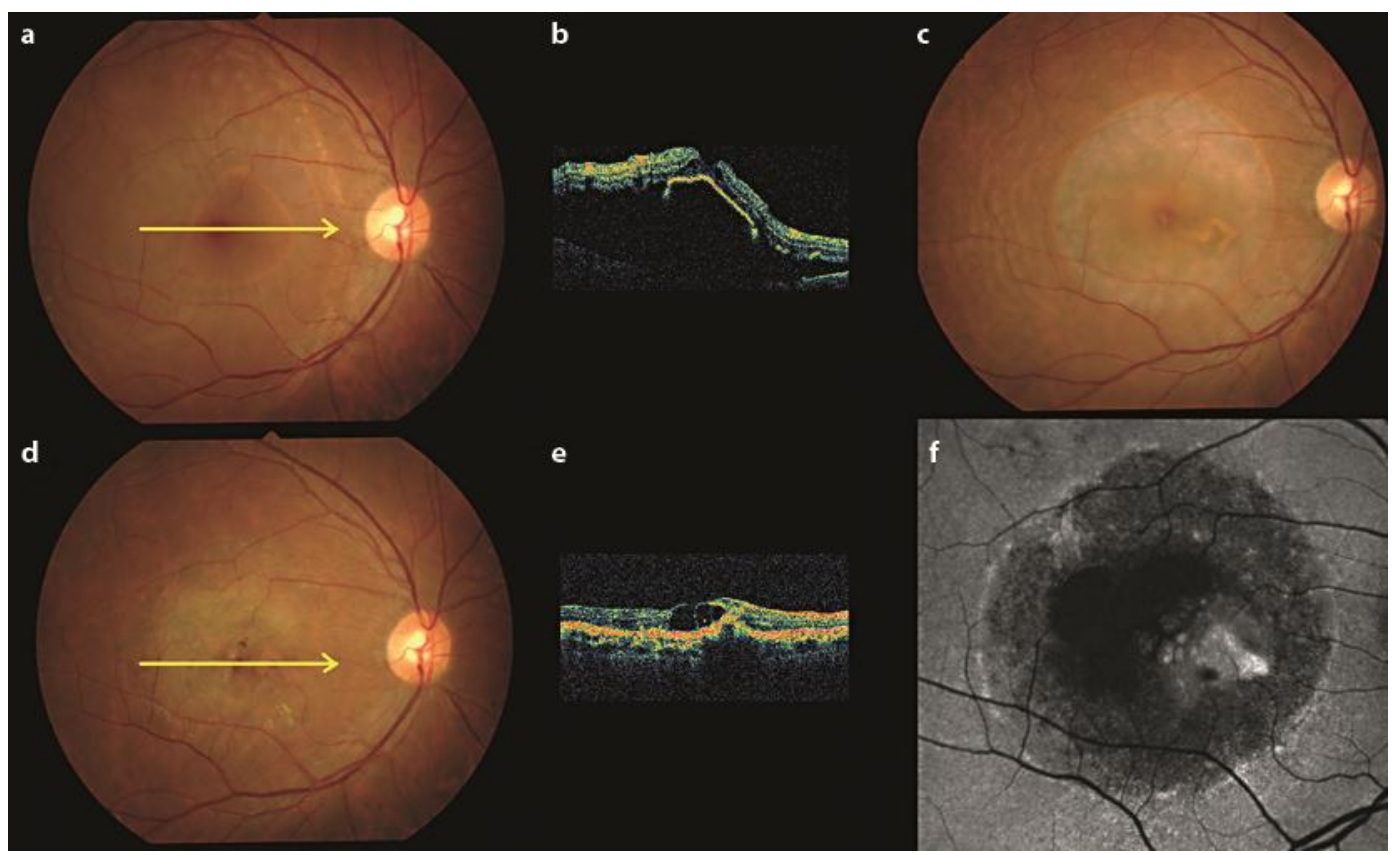

Fig. 3. Case 3. a Fundus photography of the right eye shows a $360^{\circ}$ RPE tear. b OCT demonstrates retracted RPE tissue. c Fundus photography shows partial resolution of subretinal fluid and further contracted RPE tissue adjacent to the fovea at 2-week follow-up. d-f Fundus photography shows retinal hemorrhage secondary to a small CNV within the bed of the RPE tear at 11-month follow-up. OCT confirms the presence of intraretinal fluid and repopulation of retinal pigment epithelium, and FAF imaging shows variation in hyperfluorescence patterns.

\section{References}

1 Bressler NM, Finklestein D, Sunness JS, Maguire AM, Yarian D: Retinal pigment epithelial tears through the fovea with preservation of good visual acuity. Arch Ophthalmol 1990;108:1694-1697.

$\checkmark 2$ Roydhouse TC, Roydhouse JD: Spontaneous giant retinal pigment epithelial tear. Clin Experiment Ophthalmol 2007;35:861-862.

-3 Shanmugam MP, Bhende M: Retinal pigment epithelial tears associated with idiopathic central serous chorioretinopathy. Indian J Ophthalmol 2000;48:315-317.

-4 Lim Z, Wong D: Retinal pigment epithelial rip associated with idiopathic central serous chorioretinopathy. Eye (London) 2008;22:471-473.

-5 Parchand S, Gupta V, Gupta A, Dogra MR: Bilateral giant retinal pigment epithelial rip in idiopathic central serous chorioretinopathy. Retina 2011;31:1977-1978.

6 Chang LK, Sarraf D: Tears of the retinal pigment epithelium: an old problem in a new era. Retina 2007;27:523-534.

7 Ishida Y, Kato T, Minamoto A, Yokoyama T, Jian K, Mishima HK: Retinal pigment epithelial tear in a patient with central serous chorioretinopathy treated with corticosteroids. Retina 2004;24:633-636.

8 Kunavisarut P, Pathanapitoon K, van Schooneveld M, Rothova A: Chronic central serous chorioretinopathy associated with serous retinal detachment in a series of Asian patients. Ocul Immunol Inflamm 2009;17:269-277.

9 Jampol LM, Weinreb R, Yannuzzi L: Involvement of corticosteroids and catecholamines in the pathogenesis of central serous chorioretinopathy: a rationale for new treatment strategies. Ophthalmology 2002;109:1765-1766.

10 Gupta P, Gupta A, Gupta V, Singh R: Successful outcome of giant retinal pigment epithelium rip in idiopathic central serous chorioretinopathy. Retina 2008;28:364-365.

11 Peiretti E, Iranmanesh R, Lee JJ, Klancnik JM, Sorenson JA, Yannuzzi LA: Repopulation of the retinal pigment epithelium after pigment epithelial rip. Retina 2006;26:1097-1099. 